\title{
Tumor driven by gain-of-function HER2 H878Y mutant is highly sensitive to HER2 inhibitor
}

\author{
Zexi Hu${ }^{1,2, *}$, Yong $\mathrm{Hu}^{1,2, *}$, Xicheng Liu ${ }^{2}$, Rongwen $\mathrm{Xi}^{2}$, Aiqun Zhang ${ }^{3}$, Deruo Liu ${ }^{4}$, \\ Qiang $\mathrm{Xie}^{5}$ and Liang Chen ${ }^{2,6}$ \\ ${ }^{1}$ College of Life Sciences, Beijing Normal University, Beijing, China \\ ${ }^{2}$ National Institute of Biological Sciences, Beijing, China \\ 3 The General Hospital of People's Liberation Army (301 hospital), Beijing, China \\ ${ }^{4}$ Department of thoracic surgery, China-Japan Friendship Hospital, Beijing, China \\ ${ }^{5}$ Fuzhou Pulmonary Hospital of Fujian, Fujian, China \\ ${ }^{6}$ National Institute of Biological Sciences, Collaborative Innovation Center for Cancer Medicine, Beijing, China \\ * Co-first author \\ Correspondence to: Liang Chen, email: chenliang@nibs.ac.cn \\ Keywords: HER2, H878Y, Transgenic mouse model, targeting therapy, combinational therapy \\ Received: May 13, $2015 \quad$ Accepted: August 08, $2015 \quad$ Published: September 08, 2015 \\ This is an open-access article distributed under the terms of the Creative Commons Attribution License, which permits unrestricted use, \\ distribution, and reproduction in any medium, provided the original author and source are credited.
}

\section{ABSTRACT}

HER2, a well established oncogenic member of EGFR family, is among the most intensely investigated kinase drug targets. In contrast to hotspot mutations of EGFR, few mutations of HER2 locate in activation loop within kinase domain. We previously reported the molecular mechanism underlying hyper kinase activity of HER2 ${ }^{\mathrm{H} 78 \mathrm{Y}}$, a mutation located in activation loop. However, its tumorigenicity in vivo and relevant therapeutics remain to be determined. Here, we report for the first time that HER $2^{\text {H878Y }}$ was tumorigenic in vivo in lung adenocarcinoma transgenic mouse model. Induced expression of $\mathrm{HER2}^{\mathrm{H} 878 \mathrm{Y}}$ in lung epithelial compartments resulted in formation of poorly differentiated lung adenocarcinoma with bronchioloalveolar carcinoma (BAC) features. Strikingly, we found that these tumors depended on continuous expression of HER2 ${ }^{\text {H878Y }}$ for maintenance. Typical HER2 downstream signaling mediators, including PLCY1, STAT5 and AKT, were hyperactivated in HER2 ${ }^{\text {H878Y }}$ driven lung tumors. More importantly, administration of HKI-272, a tyrosine kinase inhibitor (TKI), efficiently shrank HER2 ${ }^{\text {H878Y }}$ driven tumors in transgenic mouse model. Moreover, we found that combinational treatment with HKI272 and mTOR inhibitor, Rapamycin, showed a superior cytotoxicity to $\mathrm{H} 878 \mathrm{Y}$ mutant transformed cells and enhanced activity to elicit apoptosis and inhibit growth in situ in tumorous area. Our work therefore showed that HER2 ${ }^{\text {H878Y }}$ mutant was a reasonable drug target. Hence, our work supported the assessment of HKI-272/rapamycin treatment in clinical trials.

\section{INTRODUCTION}

HER2, a 185-Kd transmembrane receptor tyrosine kinase of the ErbB family, is a heterodimer partner to other ErbB family members to prolong and enhance downstream signaling with ampliative ligand specificity [1], resulting in activation of several downstream signaling pathways, including MAPK, PKC and AKT pathways. HER2 thus plays important roles in cell division, migration and differentiation at both cellular and organismal level $[1,2]$. Numerous studies reported that HER2 was amplified and overexpressed in several types of cancer, especially in breast cancer [2-4]. Consistently, overexpression of HER2 could continuously activate cellular proliferation and survival pathways, resulting in cell transformation and evasion from cell death [5]. Interestingly, HER2 was recently reported to be overexpressed in lung cancers with acquired resistance to EGFR targeted therapy that lack the 
secondary T790M mutation [6].

In addition to overexpressed status, mutations were also found to activate HER2 signaling loop and drive tumorigenesis. Bose et al. found seven HER2 somatic mutations in breast cancer lacking HER2 gene amplification [7]. These mutations strongly increased phosphorylation of HER2 downstream signaling proteins, including PLC $\gamma$ and MAPK, indicating that these are activating mutations; all of these mutations are sensitive to HER2 inhibitor HKI-272 (neratinib), including the lapatinib resistant mutation HER2 L755S [7]. Moreover, HER2 mutation (mainly G776insYVMA mutation) was found in 2 4\% lung cancer patients [8-10]. Most of these reported mutations were located in HER2 extracellular domain and kinase domain, but not in the activation loop [7], which is in contrast to several prominent oncogenic mutations, such as BRAF V600E and ALK R1275Q,moreover,the hotspot mutation, L858R [11] in EGFR locates in its activation loop.

Recently, H878Y mutation in HER2 was reported in $11 \%$ of hepatocellular carcinoma patients [12]. The mutation results in the mutant HER2 to harbor Y877/ Y878 motif in activation loop, similar to wild-type Y1007/ Y1008 in the JAK2 kinase [13]. Our previous work have shown phospho-Y878 forms a salt bridge with the adjacent R898 residue to stabilize the kinase in a permissive conformation, thus conferring an enhanced kinase activity for HER2 [14].

Despite of the previous biochemical characterization, whether H878Y mutant HER2 is tumorigenic in mouse level and relevant therapeutics remain to be determined. HER2 transgenic mouse models reported in earlier studies mainly focused on wild-type HER2 in breast cancer [2] and the recent HER2 G776insYVMA mutation in lung cancer model [10]. Here we reported a doxycycline inducible $\mathrm{H} 878 \mathrm{Y}$ transgenic mouse model. We showed that overexpression of H878Y mutant HER2 resulted in formation of poorly differentiated lung adenocarcinoma with bronchioloalveolar carcinoma (BAC) features and that tumors were dependent on continuous expression of mutant HER2 for maintenance. We further showed that tumors driven by HER2 H878Y mutant were sensitive to HKI-272. We also showed that combinational treatment with HKI-272 and Rapamycin resulted in enhanced toxicity to HER2 H878Y in vitro transformed cell lines and in vivo tumors.

\section{RESULTS}

\section{HER2 $^{\mathrm{H} 878 \mathrm{Y}}$ is sensitive to HER2 inhibitors}

HER2 is a more potent oncogene than other ErbB family members [1], and amplification of HER2 was reported in several types of cancer. Overexpression of
HER2 transforms normal mammary epithelial cell and induce breast cancer in mouse model [2]. Earlier reports by us and others showed that HER $2^{\mathrm{H} 878 \mathrm{Y}}$ (H878Y hereafter) is transforming in vitro $[14,15]$. We established stable cell lines to overexpress wild-type and H878Y mutant HER2 in two normal cell lines (NIH-3T3 and BEAS-2B) and checked their ability to form colonies in soft agar system. Consistent with our earlier report, we found that H878Y was more potent than wild-type HER2 to transform both of the cells (Supplementary Figure. 1A and 1B). In addition, these stable H878Y over-expressed cell lines showed markedly enhanced downstream signals, including phosphorylated PLC $\gamma$ and STAT5 (Supplementary Figure. 1C). These data again confirms H878Y is a gain-offunction mutation.

While wildtype HER2 overexpressed tumor cells were sensitive to HER2 inhibitors, those transformed cells by mutants like V777L, G776insYVMA and truncated isoform were less sensitive or resistant to HER2 inhibitor lapatinib $[9,16,17]$. In order to determine the sensitivity of H878Y to HER2 kinase inhibitors, we treated HER2 expressed cell lines with HKI-272 (Neratinib), an irreversible dual inhibitor of HER2 and EGFR currently tested in clinical trial. Consistent with our earlier report, short-term treatment (30 min) efficiently inhibited HER2 elicited signals (Figure 1A), suggesting that both wild-type and H878Y HER2 were sensitive to HKI-272 inhibition. Consequently, we observed reduced phosphorylation of AKT and ERK1/2, two canonical proliferation signals downstream of HER2. Meanwhile, a long-term (3 days) HKI-272 treatment dramatically inhibited proliferation of transformed cells (Figure 1B and 1C). Our data therefore demonstrated that H878Y mutation conferred higher oncogenic activity and elicited stronger downstream signals in comparison with wild-type HER2. More importantly, H878Y mutation was also sensitive to HKI272 in vitro cells.

\section{HER2 ${ }^{\mathrm{H} 878 \mathrm{Y}}$ drives formation of lung adenocarcinoma in transgenic mouse model}

Given that HER2 H878Y is a gain-of-function mutation and H878Y mutation is also found in a portion of cancer patients, it would be interested to test whether H878Y is tumorigenic in vivo. Previous research had reported that HER2 ${ }^{\mathrm{YVMA}}$ mutant could drive rapid development of lung adenosquamous with intrabronchial carcinomas in the proximal and distal brochioloalveolar locations [10]. The notable difference of mutation spot within HER2 kinase between HER2 ${ }^{\mathrm{YVMA}}$ and H878Y (HER2 ${ }^{\text {YVMA }}$ in kinase domain versus $\mathrm{H} 878 \mathrm{Y}$ in activation loop) motivated us to test tumorigenecity of H878Y mutant HER2 in vivo with transgenic mice.

We therefore utilized the inducible 'tet-on' expression system to generate tet-op $h H E R 2^{H 878 Y}$ transgenic 
mice, in a similar way with the reported HER2 $2^{\text {YVMA }}$ transgenic mice. To achieve this goal, an open reading frame (ORF) encoding human H878Y HER2 was cloned in between a TetO promoter/ $\beta$-globin intron and a SV40 polyA cassette (Supplementary Figure. 2A). The linear DNA fragment was microinjected into $\mathrm{FVB} / \mathrm{N}$ fertilized eggs to obtain transgenic mouse founders (Supplementary Figure $2 \mathrm{~B})$. The resulting tet-op $h H E R 2^{H 878 Y}$ founders were then crossed with CC10-rtTA mouse to generate bitransgenic mouse for specific overexpression of H878Y transgene within lung epithelium in a doxycyclineinducible manner [18]. We had successfully applied this system in several of our studies and found that this system was capable of tight control of the expression of the transgene $[19,20]$. Moreover, targeted therapy developed based on this system was widely applicable to various types of tumors, but not limited to lung cancer study [21, 22]. Among 10 available transgenic mouse founder lines, CC10-rtTA/tet-op hHER2 $2^{\text {H878Y }}$ pups of three founder lines efficiently developed lung tumors when fed on doxycycline (Dox hereafter)-containing diet for about 10 days. All of those mouse eventually died after Dox induction for around 2.5 months. After Dox treatment for one month, we sacrificed the mouse to examine the pathology of the lungs. We detected poorly differentiated lung adenocarcinomas with features of diffused bronchioloalveolar carcinoma in $\mathrm{H} 878 \mathrm{Y}$ transgenic mouse (Supplementary Figure 2C), which was in strikingly contrast to those seen in HER2 ${ }^{\mathrm{YVMA}}$ transgenic mice (glandular and squamous differentiation pathology with in situ tumor mainly located in proximal and distal airway epithelia [10]). These results were also confirmed with thorough analysis of the pathology of lungs from mouse fed on Dox for around 2 months (Figure. 2A), suggesting

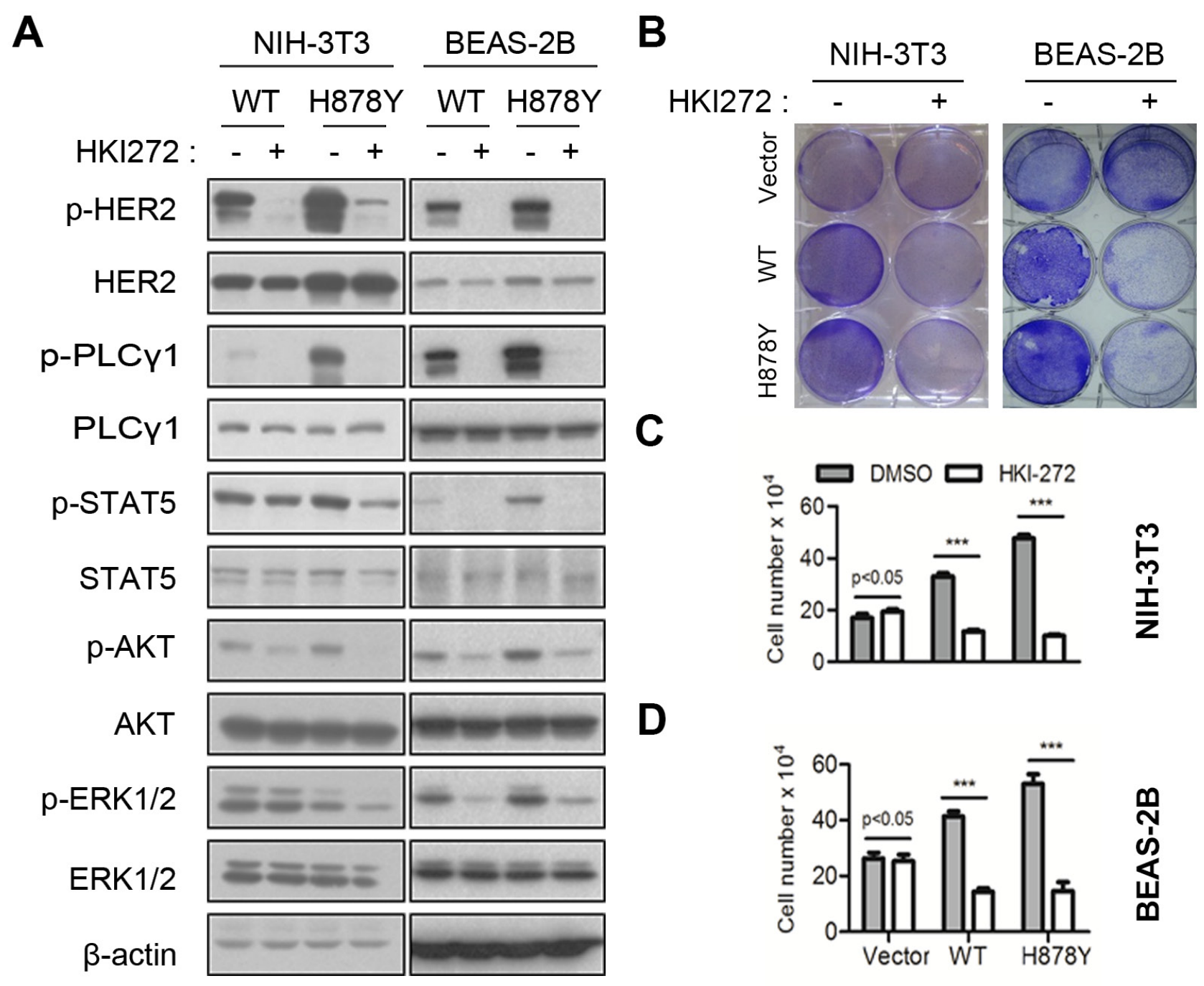

Figure 1: HER2 H878Y is sensitive to HKI-272. A. HER2 stable cell lines were treated with $500 \mathrm{nM}$ HKI-272 for 30 minutes, and then subjected to immunoblotting for detecting HER2 downstream signalings. B., C. and D. HER2 stable cell lines were treated with 500nM HKI-272 for 3 days, cells were fixed with $4 \%$ PFA and then stained with $0.01 \%$ crystal violet B., or digested to count cell numbers C. and D.. ${ }^{* * *} P<0.001$. 
different mechanisms underline tumorigenesis driven by H878Y and YVMA mutant HER2 in vivo. In addition, bitransgenic pups of all three H878Y founder lines had a medium survival below 10 weeks after Dox induction (Figure. 2B). Additionally, Immunoblotting of lung tissue lysates from tumor bearing transgenic mouse had been detected with activated HER2 downstream signals (Figure $2 \mathrm{C}$ ), consistent with the result from in vitro cell lines.

\section{H878Y driven lung tumors are dependent on continuous expression of transgene for maintenance}

Addiction to driver oncogene was reported in various types of cancers. Thus, we assessed whether H878Y induced tumors were dependent on continuous H878Y expression for tumor maintenance. We generated a cohort of bitransgenic mouse and fed them with Dox to induce lung cancer for one month. The tumor burden was documented through CT imaging (Left panel, Figure

A

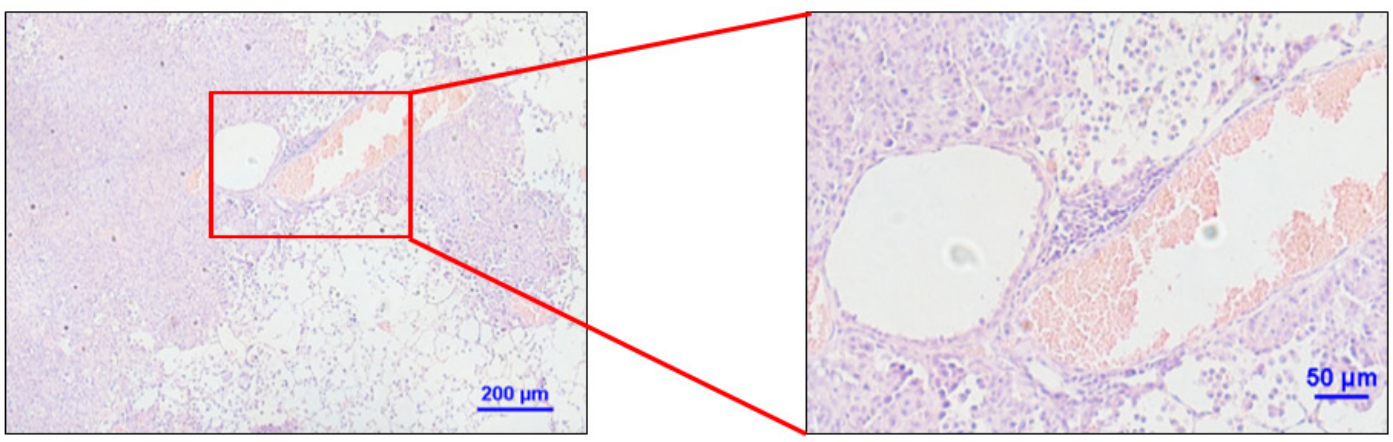

B
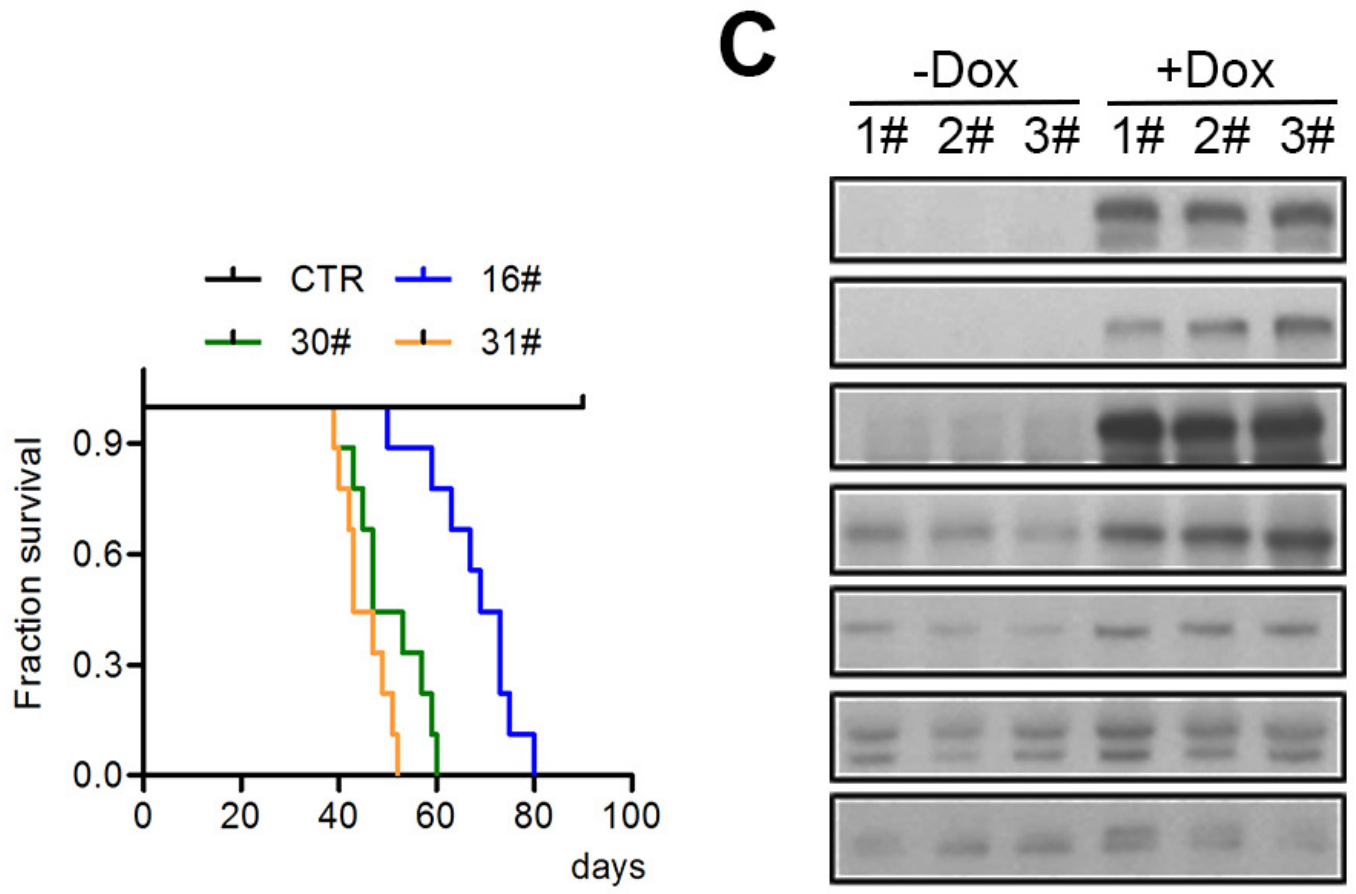

$p-H E R 2$

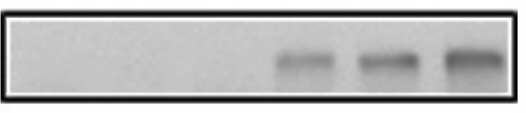

HER2

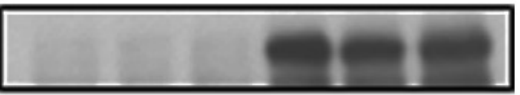

p-PLCY1

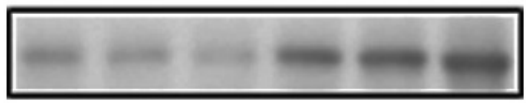

p-STAT5

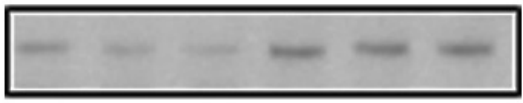

$\mathrm{p}-\mathrm{AKT}$

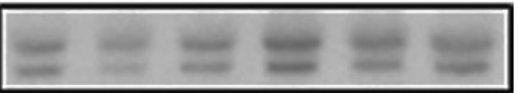

$p-E R K 1 / 2$

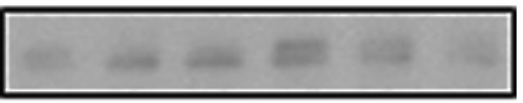

p-S6

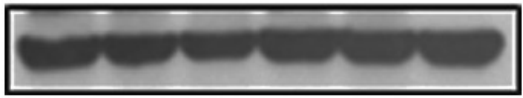

\section{$\beta$-actin}

Figure 2: H878Y mutant HER2 is tumorigenic in vivo. A. A representative H\&E image of lung tumor of H878Y transgenic mice. Scale bar: $200 \mu \mathrm{m}$ for left panel, $50 \mu \mathrm{m}$ for right panel. B. Kaplan-Meier survival curves for three different H878Y transgenic mouse founders. 16\#, 30\# and 31\# had median survival latency of 69, 47 and 43 days respectively. $n=9$ mouse for each founder line. C. H878Y bitransgenic mice were treated with doxycycline-containing or normal food for 4weeks, and then lung tissues were collected for immunoblotting of HER2 related signaling. Samples were from three different offspring of 31\# H878Y founder line. 
3A). Strinkingly, we observed that these lung tumor completely regressed in response to withdrawal of Dox for 2 weeks (middle panel, Figure 3A), indicating that H878Y continuous expression was essential for tumor maintenance. Histology analysis revealed largely normal lung, with occasional areas of thickened alveolar wall and fibrosis (right panel, Figure 3A), indicating remodeling of lung tissues.
A

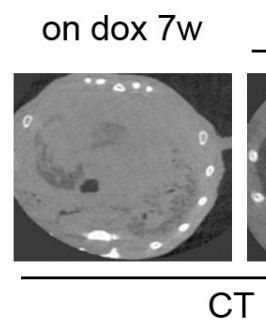

C

On/Off (days) 0/0

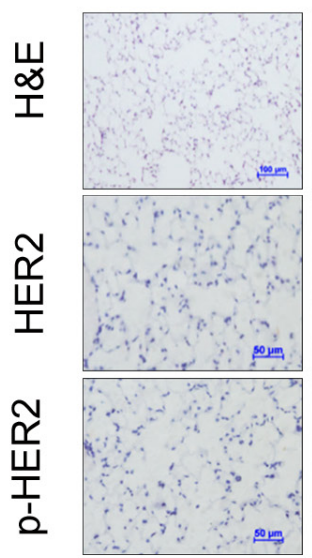

D

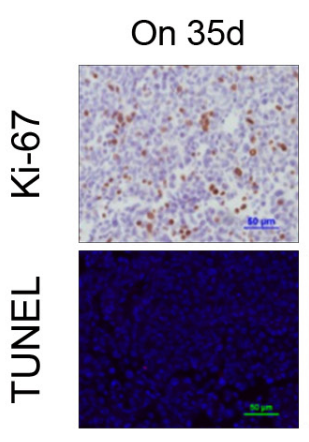

B

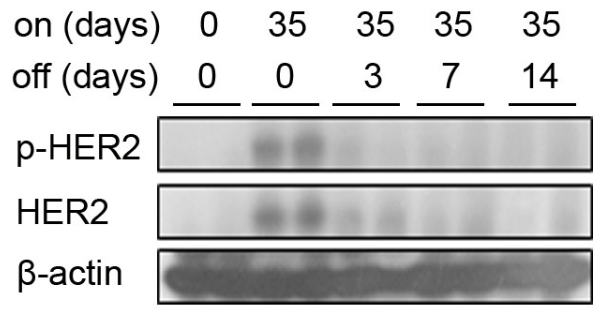

H\&E

$35 / 0$
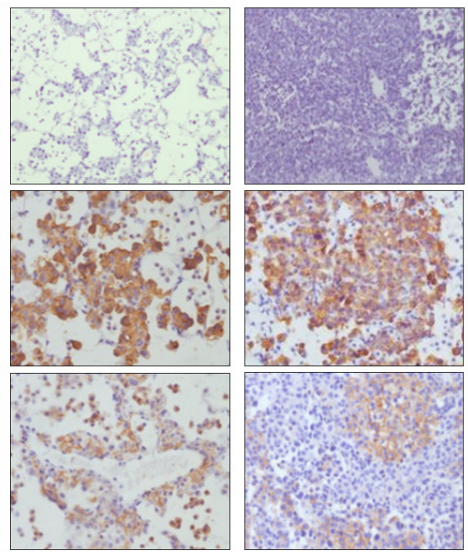

E

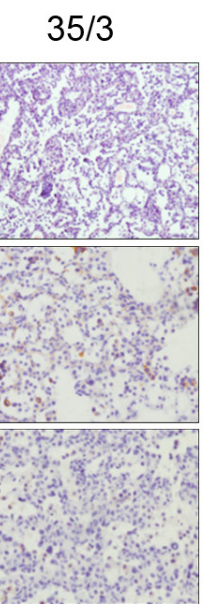

$35 / 7$

$35 / 14$
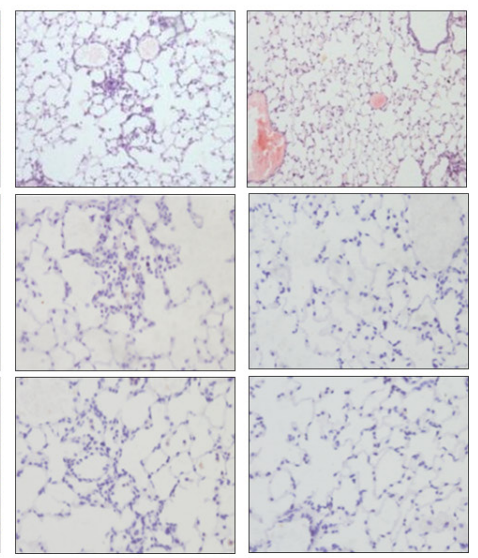

F
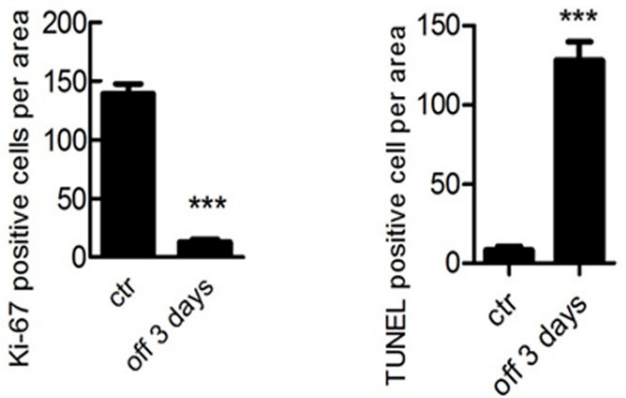

Figure 3: HER2 H878Y driven lung tumor depends on continuous expression of transgene for maintenance. A. Doxycycline withdrawal for 14 days resulted in a complete tumor regression in HER2 H878Y mice. Images showed CT scanning of one representative mouse that continuously received 7 weeks of doxycycline diet, and then underwent doxycycline withdrawal for another 14 days (left panels). H\&E staining showed no evident tumor in the lung of mice with doxycycline withdrawal (right panel). B. Immunoblotting analysis of HER2 and phos-HER2 expression in lungs of H878Y bitransgenic mice. Representative data were derived from offspring of 31\# H878Y founder line fed on normal food or on doxycycline food for the indicated days. C. Kinetics of HER2 and phosphorylated HER2 expression were revealed in in situ tumor nodules. Scale bar for H\&E was $100 \mu \mathrm{m}$ and IHC was 50 $\mu$ m. D. Ki-67 staining (top panel) and TUNEL staining (bottom panel) on H878Y bitransgenic mice on doxycycline food for 35 days and bitransgenic mice on doxycycline food for 35 days and then subjected to doxycycline withdrawal for 3 days revealed dramatically decreased in Ki-67-positive cells and increased in TUNEL-positive cells after doxycycline withdrawal. E. Quantification of Ki-67 positive cells. Data of six random tumor-containing fields were counted and analyzed. Values were mean \pm SEM $(n=6) . * * P<0.01$. F. Quantification of TUNEL positive cells. Data of six random tumor-containing fields were counted and analyzed. Values were mean $\pm \operatorname{SEM}(n=6) . * *<0.01$. 
Next, we wondered whether discontinuing HER2 expression led to a reduction of HER2 signal. To validate our hypothesis, we analyzed HER2 expression and function in these mouse at different time points. We collected lung tissues from mouse fed on Dox for 35 days, on Dox 35 days/off Dox for 3, 7, or 14 days serial treatment as well as normal diet treatment, and analyzed HER2 and phospho-HER2 expression through Western blotting. We found that HER2 signal arose from undetectable in the mouse fed on normal diet to strong expression level in mouse on continuous Dox for 35 days, and rapidly waned after Dox withdrawal (Figure 3B). To validate these phenomena in situ in tumor tissues, we analyzed HER2 and phospho-HER2 levels in lungs through immunohistochemistry (IHC). Lung tissues from bitransgenic mouse without Dox induction were negative of HER2 and phospho-HER2 signals. In contrast, we detected strong HER2 and phospho-HER2 activated signals in mouse fed on Dox for 25 days. After 35 days Dox induction, strong HER2 and phosphoHER2 signals were detected in poorly differentiated lung adenocarcinoma in the bitransgenic mouse. Strikingly, withdrawal of Dox for just 3 days led to regression of HER2 signals to the baseline level, and remained undetectable by 7 and 14 days after Dox withdrawal (Figure 3C).

To determine the biological mechanism underlying tumor regression, we assessed the cellular proliferative/ apoptotic index in the tumor regions of mouse after withdrawing Dox. As short as for 3 days of Dox withdrawl, Ki-67, a classical cell proliferation marker, was dramatically downregulated in tumors (Figure 3D,

A

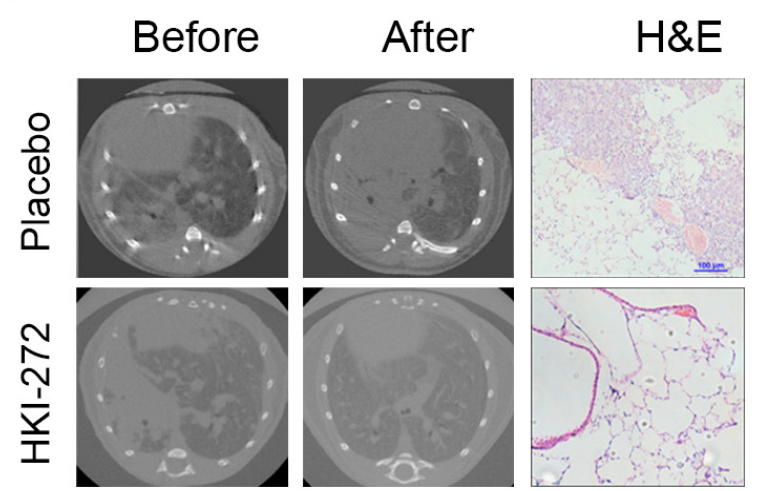

C
B

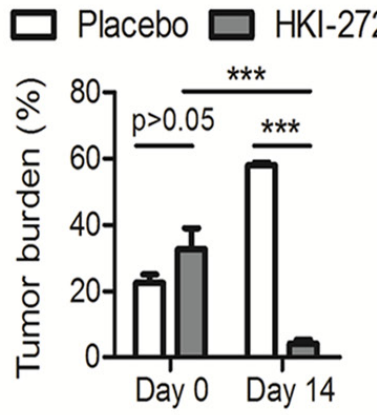

D
$\mathbf{E}$

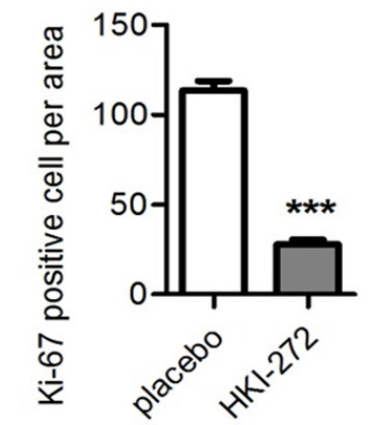

$\mathbf{F}$
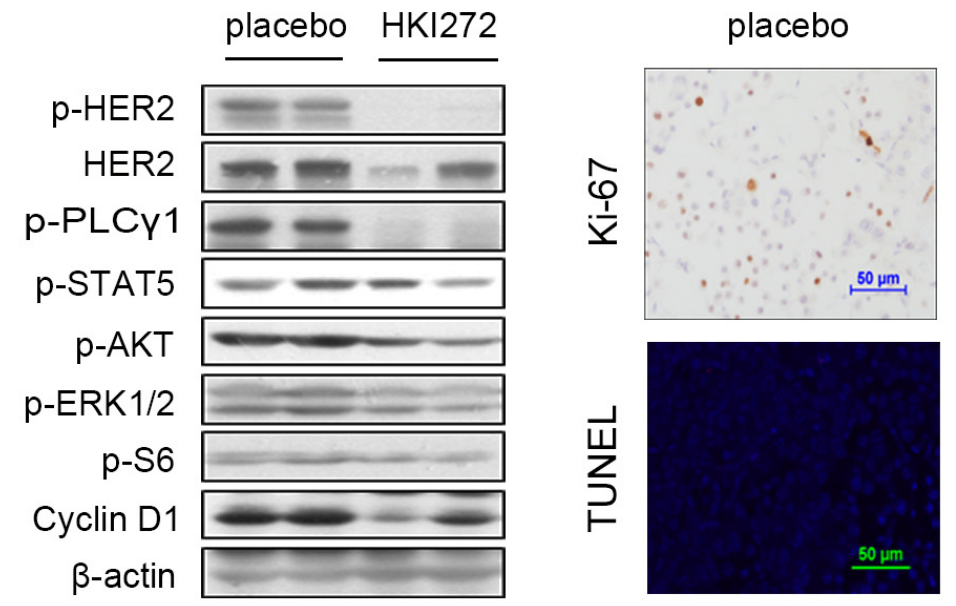

$\mathrm{HKI} 272$
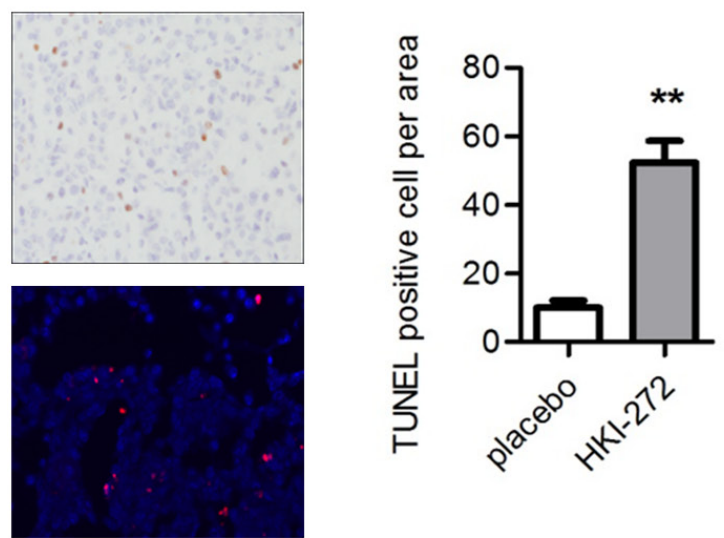

Figure 4: HER2 H878Y-driven lung tumor is sensitive to HKI-272 treatment. A. HKI-272 treatment led to regression of lung tumor in transgenic mice. Mice were fed with doxycycline food for 4 weeks, and then treated with vehicle or HKI-272 at $50 \mathrm{mg} / \mathrm{kg}$ for 2 weeks respectively. Three mouse of each treatment were imaged with CT. Scale bar for H\&E picture, $100 \mu \mathrm{m}$. B. Quantification of tumor areas shown in A.. ImageJ software was used to determine tumor areas in the CT images and the percentage of tumor areas against the whole lung was calculated. Values were mean $\pm \operatorname{SEM}(n=3)$. ${ }^{* * *} P<0.001$. C. Immunoblotting analysis of lung tissues from H878Ydriven tumors. HER2 H878Y mice were treated with vehicle and HKI-272 for three days. Lung tissues were analyzed through western blot. D. Representative photographs of cross-sectional tumors assayed for Ki-67 and TUNEL from mouse mentioned in C.. Scale bars, 50 $\mu$ m. E., F. Quantification of Ki-67 positive cells E. and TUNEL positive cells F. Values were mean $\pm \operatorname{SEM}(n=4)$. ${ }^{* *} P<0.01, * * * P<0.001$. 
top panel; quantification in Figure 3E). Conversely, the number of apoptotic cells within tumor were increased by TUNEL (terminal deoxynucleotidyl transferase dUTP nick-end labeling) assay (Figure 3D, bottom panel; quantification in Figure 3F).

Taken together, these data showed that H878Ydriven tumors required the continuous function of HER2 mutant for tumor maintenance, suggesting that targeted HER2 H878Y signaling maybe a valid option.

\section{hHER2 ${ }^{\mathrm{H} 878 \mathrm{Y}}$-driven tumors are sensitive to $\mathrm{HKI}-$ 272 treatment in vivo}

We have shown that HKI-272 exhibited superior inhibition of HER2 elicited signaling in vitro cells [14]. Studies from our transgenic mouse model showed that H878Y mutant HER2 function was important for the maintenance of tumor. To mimic clinical settings, we tested whether HKI-272 was effective in shrinking lung adenocarcinoma in transgenic mouse model. We fed a cohort of CC10-rtTA/tet-op hHER $2^{H 878 Y}$ mouse with Dox diet for one month and documented the tumor burden through CT imaging. Strikingly, we found that HKI-272 treatment for 2 weeks led to almost complete regression of the tumors, while tumors in placebo-treated group grew rapidly (Figure 4A,top two panels for CT images; Figure 4B for quantification). Histology data were consistent with imaging (Figure 4A, lower panel).

To see the effect of HKI-272 on HER2 downstream signals, we conducted immunoblotting analysis on the lung tissues. As expected, we found that HER2 downstream mediators, including PLC $\gamma$, STAT5, AKT and Cyclin D1, were decreased in tumor tissues lysates (Figure 4C). We further checked whether HKI-272 treatment led to apoptosis of tumor cells. As readouts for therapeutic efficacy, we evaluated proliferative (Ki-67) and apoptotic (TUNEL) marker in situ in the tumor cells by IHC. HKI272 treatment for 3 days was effective in inhibiting tumor cell growth, as indicated by $\mathrm{Ki}-67$ staining, and HKI-272 also eliciting tumor cell apoptosis, as indicated by TUNEL staining (Figure 4D for images; Figure 4E and 4F for quantification).

\section{Combinational treatment with HKI-272 and Rapamycin shows enhanced cytotoxicity to tumor driven by hHER2 ${ }^{\mathrm{H} 878 \mathrm{Y}}$}

Although HKI-272 is effective in shrinking H78Y mutant HER2 driven tumors, tumors in patients may be more complicated and combinational therapy may be necessary in those cases. The inhibitors preferably target on the same protein $[23,24]$, or distinct signaling components in the same pathway [25]. This was particularly relevant due to the release of feedback inhibition during targeting ErbB family members [26].
We therefore tested whether combinational therapy could enhance the cytotoxicity to tumors.

Of note, although we observed a reduction of AKT phosphoryation in HER2 transformed cells in both NIH3T3 and BEAS-2B cellular background after treatment with HKI-272, residual phosphorylation of AKT signal was still to be detected (Figure 1A). AKT was an important effector for cell survival. Complete inhibition of AKT signaling in tumor cells would be ideal. Interestingly, the inhibition of the PI3K-Akt-mTOR signaling pathway with a PI3K and mTOR dual inhibitor (NVP-BEZ235) was shown to be effective in reducing viable HER2-driven cancer cells [27]. This suggests that Rapamycin might represent a suitable candidate for an adjuvant therapeutic modality that can be applied in combination with HKI-272 for treating $\mathrm{H} 878 \mathrm{Y}$ mutant-driven cancers.

We found that Rapamycin alone elicited a moderate growth inhibition in $\mathrm{H} 878 \mathrm{Y}$ mutant transformed $\mathrm{Ba} /$ F3 cells, but not in WT HER2 transformed cells (Supplementary Figure 3), further suggesting that the increased AKT signaling elicited by the H878Y mutant rendered cells more sensitive to mTOR pathway inhibition. To further evaluate the potency of combinational treatment with HKI-272 and Rapamycin, we assessed the $\mathrm{IC}_{50}$ values of combined therapy with HKI-272 and Rapamycin compared to HKI-272 monotherapy. The $\mathrm{IC}_{50}$ of HKI-272 alone was approximately $2.4 \mathrm{nM}$ and shifted a decrease to approximately $0.3 \mathrm{nM}$ in the presence of $2 \mathrm{nM}$ Rapamycin (Figure 5A). This finding showed that rapamycin synergized with HKI-272 to effectively kill H878Y HER2 mutant-transformed $\mathrm{Ba} / \mathrm{F} 3$ cells. Consistently, we found that combinational treatment with Rapamycin and HKI272 led to a decrease in signals of S6 phosphorylation and Cyclin D1 as well as inhibition of HER2, AKT and ERK phosphorylation in H878Y HER2 transformed 3T3 cells (Figure 5B). Our data showed that combined treatment with HKI-272 and rapamycin resulted in superior inhibition of signaling elicited by H878Y HER2 compared to either agent alone.

We further tested the combinational therapy in vivo. We treated a cohort of tumor-bearing bitransgenic mouse with vehicle, Rapamycin, HKI-272 and Rapamycin/HKI272 combination. Rapamycin treatment for 3 days was effective in inhibiting tumor cell growth, as indicated by Ki-67 staining, but showned no evident effect in eliciting tumor cell death, as indicated by TUNEL staining (Figure 5C-5E). However, HKI-272 alone was effective both in tumor cell growth inhibition and apoptosis induction. Strikingly, we found that HKI-272/Rapamycin combinational therapy elicited significantly increased tumor cell death and inhibited cellular proliferation compared to either drug alone (Figure 5C-5E). These results showed that the combinational treatment was more toxic to tumor cells transformed by HER2 H878Y in vivo. 

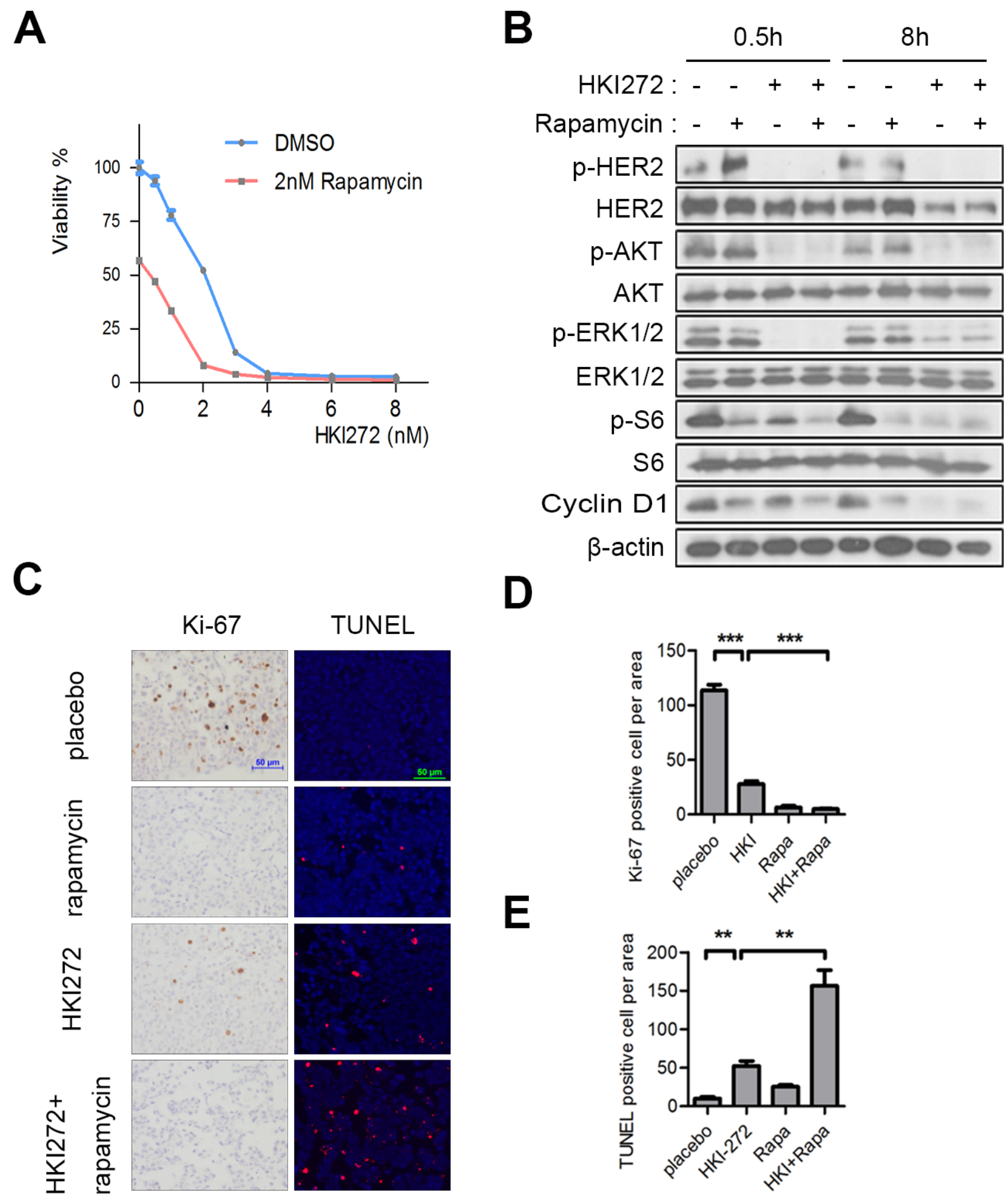

Figure 5: Rapamycin enhances the efficacy of HKI-272 towards HER2 H878Y-driven tumors. A. H878Y transformed Ba/ f3 cells were treated with HKI-272 alone or a combination of HKI-272 and 2nM Rapamycin for 3 days. Cell viability was determined by CellTiter-Glo assay. Values were mean $\pm \operatorname{SEM}(n=6)$. B. 3T3-H878Y cells were treated with DMSO, 500nM Rapamycin, 500nM HKI272 or a combination of Rapamycin and HKI-272 for indicated time, and then subjected to immunoblotting analysis C.Representative photographs of cross-sectional tumors assayed for Ki-67 and TUNEL from treated mice. HER2 H878Y bitransgenic mouse were treated with vehicle, HKI-272, Rapamycin, or the combinational HKI-272/ Rapamycin for 3 days respectively. Scale bars, 50 $\mu \mathrm{m}$. D.,E.Quantification of Ki-67 positive cells D. and TUNEL positive cells E. in the mouse C. Values were mean \pm SEM $(n=4)$. HKI, HKI-272; Rapa, Rapamycin. $* * P<0.01, * * * P<0.001$ 


\section{DISCUSSION}

Due to its high rate of deregulation (either overexpression or mutation) in various tumor types, HER2 is among the most intensely investigated drug targets. Mutations of HER2 within kinase domain are roughly grouped into two categories: activation loop and nonactivation-loop mutations. $\mathrm{H} 878 \mathrm{Y}$ is the only mutation located within activation loop reported for HER2. Our previous and present work have shown that H878Y HER2 is a gain-of-function mutation, which is tumorigenic in vivo in transgenic mouse model. More importantly, the tumor driven by this oncogene is dependent on its continuous expression for maintenance and these tumors can be effectively treated by a HER2 kinase inhibitor in this mouse model.

Activation loop of EGFR is frequently detected to be mutated in a variety of tumors. For example, L858R is one of the most potent mutations found in lung cancer patients [11]. These mutations are reported to enhance kinase activity of EGFR. Many researches have reported that these mutations are tumorigenic in vivo and that these mutations sensitize the tumor to EGFR TKI treatment. In contrast, $\mathrm{H} 878 \mathrm{Y}$ is the only mutation reported in activation loop of HER2. We recently reported that H878Y was a gain-of-function mutation in vitro cell lines, and revealed a potential mechanism underlying this gain-of-function. Interestingly, we found $\mathrm{H} 878 \mathrm{Y}$ mutation similar to L858R in EGFR in the way that it was a TKI-sensitizing mutation, which was different from 776 insertional mutation HER2 ${ }^{\text {YVMA }}$. In our present work, we found that HKI-272, now entering clinic trial, was effective in treating H878Y mutant HER2 driven tumors. However, we had found that HKI-272 alone was not as effective as in H878Y case for treating tumors driven by HER $2^{\mathrm{YVMA}}$ (data not shown).

Oncogenic HER2 was reported to activate some downstream effectors, such as PLC $\gamma$ [28]. Considering the difference in locations within HER2 protein, the difference in the phenotypes of formed tumors, and the difference in drug sensitivity revealed in our work, HER2 H878Y and HER2 ${ }^{\mathrm{YVMA}}$ mutations may impact HER2 function in drastically different ways. Our data therefore highlighted the necessity and importance to further study the difference in signaling between H878Y and most other non-activatioin-loop-localizing mutations (such as HER2 ${ }^{\mathrm{YVMA}}$ ).

A number of HER2 inhibitory reagents are now available: small molecules and antibodies. Our earlier work reported that second generation inhitors such as BIBW-2992 and HKI-272 outperformed reversible inhibitors against HER2 transformed $\mathrm{Ba} / \mathrm{F} 3$ cells in two ways: HKI-272 was less toxic to nontransformed cells, and HKI-272 was more potent in killing HER2 transformed cells. Antibodies can inhibit homo-dimerization of HER2 or hetero-dimerization with other members of ErbB family. Our work had reported that exon19 deletion mutant EGFR in lung cancers was not dependent on dimerization for functioning, and therefore, antibodies may be less effective on these mutations [29]. In the case of TKI resistance, combinational treatment with antibody and TKI is expected to kill HER2 transformed cells more effectively [30].

Of note, mTOR inhibition could upregulate the autophage. We noted in our mouse model that rapamycin synergized with HKI-272 to induce autophagy in H878Y driven lung tumors (data not shown).

Tumors in patients developed through long terms of inactivation of tumor suppressor genes and activation of growth promoting oncogenes, such that treatments that effectively killed tumor cell cell lines or shrank tumors in mouse models sometimes fail in patients. Combinational treatments based on reasonably designed schemes are preferable. We found that $\mathrm{H} 878 \mathrm{Y}$ drove stronger activation of AKT signaling and residual AKT activation remained to be detected after HKI-272 treatment. We therefore tested Rapamycin, an inhibitor to block the mTOR signaling downstream of AKT to combine HKI-272 as a therapeutic strategy. We had confirmed the superior treatment effect in cell lines and in transgenic mouse models with this combined therapy, which caused tumor cell growth inhibition and apoptosis induction for HER2 H878Y mutant.

Our current work therefore revealed that H878Y mutant was tumorigenic in vivo and was a reasonable target for tumors driven by this HER2 mutation. Combinational treatment with HKI-272 and Rapamycin is effective in vivo mouse models, and warrant further validation in clinic.

\section{MATERIALS AND METHODS}

\section{Mouse work}

All mice were housed in a pathogen-free environment at the National Institute of Biological Sciences, Beijing (NIBS). All experimental protocols were approved by the Institutional Committee for Animal Care and Use at NIBS. All animal work was performed in accordance with the approved protocol.

\section{Cell lines}

All cell lines used in this study were purchased from ATCC (American Type Culture Collection) and cultured in Dulbecco's Modified Eagle's Medium (DMEM) containing $10 \%$ FCS (for NIH-3T3, referred to as 3T3) or $10 \% \mathrm{FBS}$ (for BEAS-2B). Ba/f3 cells were maintained in RPMI1640 medium containing 10\%FBS and IL-3. Cell culture media were supplemented with $10 \mathrm{mM}$ glutamine and $1 \%$ penicillin and streptomycin and incubated at $37^{\circ} \mathrm{C}$ 
in a $5 \% \mathrm{CO}_{2}$ incubator.

\section{Sable cell line generation, Soft agar and cell viability assay}

Plasmids constructs, stable cell lines generation, soft agar assay and cell viability assay were described as previous study [14].

\section{Western blotting}

All cells or tissues were lysed in RIPA buffer (Beyotime) supplemented with protease and phosphatase inhibitors (Roche). Following lysis, the cells were sonicated. Western blotting was performed using standard methods. Antibody against $\beta$-actin was from Sigma, other antibodies were purchased from Cell Signaling Technology.

\section{Generation of the CC10-rtTA/tet-op- hHER2H878Y mouse cohort}

To generate Tet-op-hHER2 $2^{H 878 Y}$ transgenic mice, human HER2 H878Y DNA was subcloned into pTRETight (Clonetech), and then a $\beta$ - globin intron was subcloned into this plasmid and just located between the Tet-responsive $\mathrm{P}_{\text {tight }}$ promoter and H878Y ORF. The DNA fragments containing Tet-op- $\beta$-globin intronhHER2 H878Y-SV40 polyA were injected into FVB/N blastocysts. The progeny were genotyped as described [26]. All mouse were housed in SPF room at NIBS' animal room and crossed with CC10-rtTA [26]. Genotyping of CC10-rtTA and tet-op-hHER2 $2^{H 878 Y}$ were performed by using following primers: for $C C 10-r t T A$, forward primer: AAAATCTTGCCAGCTTTCCCC ; reverse primer: ACTGCCCATTGCCCAAACAC. For tet-op- $h H E R 2^{H 878 Y}$, forward primer: GGCACCCAGCTCTTTGAGGA ; reverse primer: CACCTCTCGCAAGTGCTCCA .

\section{Drug treatment in vivo and micro-CT}

All mice were housed in a pathogen-free environment at the National Institute of Biological Sciences, Beijing (NIBS). All mouse were handled strictly in accordance with good animal practice as defined by the Animal Facility in NIBS, and all animal work were done with approval from Institutional Committee for Animal Care and Use in NIBS. CC10-rtTA/Tet-op-hHER2 $2^{\text {H878Y }}$ mouse fed on Dox food when the pups reached 30 days old. After continuous exposure to doxycycline-containing diets (Research Diets) for 5 weeks, mouse were then randomized to groups and treated daily with $50 \mathrm{mg} / \mathrm{kg}$ HKI-272 (Selleck Chemicals) or $2 \mathrm{mg} / \mathrm{kg}$ Rapamycin (Selleck Chemicals). HKI-272 was formulated in $0.5 \%$ methocellulose- $0.4 \%$ Tween 80 and was administered by oral gavage, and Rapamycin formulated in 5\%-PEG 400$5 \%$ Tween 80 was administrated by i.p. injection. MicroCT analysis was performed using the Siemens Inveon PET-CT analyzer.

\section{H\&E and IHC staining}

Lungs were collected from animals and fixed overnight in $10 \%$ formalin supplied with $1 \mathrm{mM} \mathrm{NaF}$, $1 \mathrm{mM} \mathrm{NaVO}$, 1mM PMSF. Hematoxylin and eosin ( H\&E) staining and Immuno- histological (IHC) staining were performed on $5 \mu \mathrm{m}$ formalin-fixed paraffin sections. The antibodies used were described in Western Blotting section. The ABC-Elite Rabbit IgG kit (Vector Laboratories) was used as secondary detection system (45min incubation at room temperature). DAB (Sigma) was used as a substrate. Ki-67 antibody (Vector Laboratories) was used to assess the proliferation of cells. Apoptosis was measured by TUNEL assay with TMR red In Situ Cell Death detection Kit (Roche) according to the manual. The nucleus was stained with DAPI.

\section{ACKNOWLEDGMENTS}

This work was funded by National Natural Science Foundation of China grant 81472606 , Chinese Ministry of Science and Technology grant (973 grant) 2011CB812401, and the Beijing Municipal Government.

\section{Author contributions}

L.C. designed and supervised experiments. Z.H., Y.H. and X.L. performed experiments and data analysis. R.X., A.Z., D.L., Q.X., and L.C. contributed to data analysis. L.C. wrote the manuscript with contributions of all authors.

\section{CONFLICTS OF INTERESTS}

The authors declare no competing financial interests.

\section{REFERENCES}

1. Yarden $\mathrm{Y}$ and Sliwkowski MX. Untangling the ErbB signalling network. Nat Rev Mol Cell Biol. 2001; 2:127137.

2. Moasser MM. The oncogene HER2: its signaling and transforming functions and its role in human cancer pathogenesis. Oncogene. 2007; 26:6469-6487.

3. Yu D and Hung MC. Overexpression of ErbB2 in cancer and ErbB2-targeting strategies. Oncogene. 2000; 19:61156121. 
4. Moasser MM. Targeting the function of the HER 2 oncogene in human cancer therapeutics. Oncogene. 2007; 26:65776592.

5. Hynes NE and MacDonald G. ErbB receptors and signaling pathways in cancer. Curr Opin Cell Biol. 2009; 21:177-184.

6. Takezawa K, Pirazzoli V, Arcila ME, Nebhan CA, Song X, de Stanchina E, Ohashi K, Janjigian YY, Spitzler PJ, Melnick MA, Riely GJ, Kris MG, Miller VA, Ladanyi M, Politi K and Pao W. HER2 amplification: a potential mechanism of acquired resistance to EGFR inhibition in EGFR mutant lung cancers that lack the second-site EGFR T790M mutation. Cancer Discovery. 2012;2:922-33

7. Bose R, Kavuri SM, Searleman AC, Shen W, Shen D, Koboldt DC, Monsey J, Goel N, Aronson AB, Li S, Ma CX, Ding L, Mardis ER and Ellis MJ. Activating HER2 Mutations in HER2 Gene Amplification Negative Breast Cancer. Cancer Discovery. 2012; 3:224-237.

8. Stephens P, Hunter C, Bignell G, Edkins S, Davies H, Teague J, Stevens C, O'Meara S, Smith R, Parker A, Barthorpe A, Blow M, Brackenbury L, Butler A, Clarke O, Cole J, et al. Lung cancer: intragenic ERBB2 kinase mutations in tumours. Nature. 2004; 431:525-526.

9. Wang SE, Narasanna A, Perez-Torres M, Xiang B, Wu FY, Yang S, Carpenter G, Gazdar AF, Muthuswamy SK and Arteaga CL. HER2 kinase domain mutation results in constitutive phosphorylation and activation of HER2 and EGFR and resistance to EGFR tyrosine kinase inhibitors. Cancer Cell. 2006; 10:25-38.

10. Perera SA, Li D, Shimamura T, Raso MG, Ji H, Chen L, Borgman CL, Zaghlul S, Brandstetter KA, Kubo S, Takahashi M, Chirieac LR, Padera RF, Bronson RT, Shapiro GI, Greulich H, et al. HER2YVMA drives rapid development of adenosquamous lung tumors in mice that are sensitive to BIBW2992 and rapamycin combination therapy. Proc Natl Acad Sci U S A. 2009; 106:474-479.

11. Paez JG, Janne PA, Lee JC, Tracy S, Greulich H, Gabriel S, Herman P, Kaye FJ, Lindeman N, Boggon TJ, Naoki K, Sasaki H, Fujii Y, Eck MJ, Sellers WR, Johnson BE, et al. EGFR mutations in lung cancer: correlation with clinical response to gefitinib therapy. Science. 2004; 304:14971500.

12. Bekaii-Saab T, Williams N, Plass C, Calero MV and Eng C. A novel mutation in the tyrosine kinase domain of ERBB2 in hepatocellular carcinoma. BMC Cancer. 2006; 6:278.

13. Feng J, Witthuhn BA, Matsuda T, Kohlhuber F, Kerr IM and Ihle JN. Activation of Jak2 catalytic activity requires phosphorylation of Y1007 in the kinase activation loop. Mol Cell Biol. 1997; 17:2497-2501.

14. Hu Z, Wan X, Hao R, Zhang H, Li L, Li L, Xie Q, Wang P, Gao Y, Chen S, Wei M, Luan Z, Zhang A, Huang N and Chen L. Phosphorylation of Mutationally Introduced Tyrosine in the Activation Loop of HER2 Confers Gain-ofFunction Activity. PLoS One. 2015; 10:e0123623.

15. Kancha RK, von Bubnoff N, Bartosch N, Peschel C, Engh
RA and Duyster J. Differential sensitivity of ERBB2 kinase domain mutations towards lapatinib. PLoS One. 2011; 6:e26760.

16. Hudziak RM, Lewis GD, Shalaby MR, Eessalu TE, Aggarwal BB, Ullrich A and Shepard HM. Amplified expression of the HER2/ERBB2 oncogene induces resistance to tumor necrosis factor alpha in NIH $3 \mathrm{~T} 3$ cells. Proc Natl Acad Sci U S A. 1988; 85:5102-5106.

17. Xia W, Liu Z, Zong R, Liu L, Zhao S, Bacus SS, Mao Y, He J, Wulfkuhle JD, Petricoin EF, 3rd, Osada T, Yang XY, Hartman ZC, Clay TM, Blackwell KL, Lyerly HK, et al. Truncated ErbB2 expressed in tumor cell nuclei contributes to acquired therapeutic resistance to ErbB2 kinase inhibitors. Mol Cancer Ther. 2011; 10:1367-1374.

18. Mehrad B, Wiekowski M, Morrison BE, Chen SC, Coronel EC, Manfra DJ and Lira SA. Transient lung-specific expression of the chemokine $\mathrm{KC}$ improves outcome in invasive aspergillosis. Am J Respir Crit Care Med. 2002; 166:1263-1268.

19. Ji H, Li D, Chen L, Shimamura T, Kobayashi S, McNamara K, Mahmood U, Mitchell A, Sun Y, Al-Hashem R, Chirieac LR, Padera R, Bronson RT, Kim W, Janne PA, Shapiro GI, et al. The impact of human EGFR kinase domain mutations on lung tumorigenesis and in vivo sensitivity to EGFRtargeted therapies. Cancer Cell. 2006; 9:485-495.

20. Zhou W, Ercan D, Chen L, Yun CH, Li D, Capelletti M, Cortot AB, Chirieac L, Iacob RE, Padera R, Engen JR, Wong KK, Eck MJ, Gray NS and Janne PA. Novel mutantselective EGFR kinase inhibitors against EGFR T790M. Nature. 2009; 462:1070-1074.

21. Engelman JA, Chen L, Tan X, Crosby K, Guimaraes AR, Upadhyay R, Maira M, McNamara K, Perera SA, Song Y, Chirieac LR, Kaur R, Lightbown A, Simendinger J, Li T, Padera RF, et al. Effective use of PI3K and MEK inhibitors to treat mutant Kras G12D and PIK3CA H1047R murine lung cancers. Nat Med. 2008; 14:1351-1356.

22. De Luca A, Maiello MR, D'Alessio A, Pergameno M and Normanno N. The RAS/RAF/MEK/ERK and the PI3K/ AKT signalling pathways: role in cancer pathogenesis and implications for therapeutic approaches. Expert opinion on therapeutic targets. 2012; 16:S17-27.

23. Regales L, Gong Y, Shen R, de Stanchina E, Vivanco I, Goel A, Koutcher JA, Spassova M, Ouerfelli O, Mellinghoff IK, Zakowski MF, Politi KA and Pao W. Dual targeting of EGFR can overcome a major drug resistance mutation in mouse models of EGFR mutant lung cancer. The Journal of clinical investigation. 2009; 119:3000-3010.

24. Shen ZX, Shi ZZ, Fang J, Gu BW, Li JM, Zhu YM, Shi JY, Zheng PZ, Yan H, Liu YF, Chen Y, Shen Y, Wu W, Tang W, Waxman S, De The H, et al. All-trans retinoic acid/ As2O3 combination yields a high quality remission and survival in newly diagnosed acute promyelocytic leukemia. Proc Natl Acad Sci U S A. 2004; 101:5328-5335.

25. Lito P, Pratilas CA, Joseph EW, Tadi M, Halilovic E, Zubrowski M, Huang A, Wong WL, Callahan MK, 
Merghoub T, Wolchok JD, de Stanchina E, Chandarlapaty S, Poulikakos PI, Fagin JA and Rosen N. Relief of profound feedback inhibition of mitogenic signaling by RAF inhibitors attenuates their activity in BRAFV600E melanomas. Cancer Cell. 2012; 22:668-682.

26. Li D, Shimamura $\mathrm{T}$, Ji H, Chen L, Haringsma HJ, McNamara K, Liang M-C, Perera SA, Zaghlul S, Borgman CL, Kubo S, Takahashi M, Sun Y, Chirieac LR, Padera RF, Lindeman NI, et al. Bronchial and Peripheral Murine Lung Carcinomas Induced by T790M-L858R Mutant EGFR Respond to HKI-272 and Rapamycin Combination Therapy. Cancer Cell. 2007; 12:81-93.

27. Faber AC, Li D, Song Y, Liang MC, Yeap BY, Bronson RT, Lifshits E, Chen Z, Maira SM, Garcia-Echeverria C, Wong KK and Engelman JA. Differential induction of apoptosis in HER2 and EGFR addicted cancers following PI3K inhibition. Proc Natl Acad Sci U S A. 2009; 106:1950319508.

28. Peles E, Levy RB, Or E, Ullrich A and Yarden Y. Oncogenic forms of the neu/HER2 tyrosine kinase are permanently coupled to phospholipase C gamma. EMBO J. 1991; 10:2077-2086.

29. Cho J, Chen L, Sangji N, Okabe T, Yonesaka K, Francis JM, Flavin RJ, Johnson W, Kwon J, Yu S, Greulich H, Johnson BE, Eck MJ, Janne PA, Wong KK and Meyerson M. Cetuximab response of lung cancer-derived EGF receptor mutants is associated with asymmetric dimerization. Cancer Res. 2013; 73:6770-6779.

30. Janjigian YY, Smit EF, Groen HJ, Horn L, Gettinger S, Camidge DR, Riely GJ, Wang B, Fu Y, Chand VK, Miller VA and Pao W. Dual inhibition of EGFR with afatinib and cetuximab in kinase inhibitor-resistant EGFR-mutant lung cancer with and without T790M mutations. Cancer Discov. 2014; 4:1036-1045. 